\title{
Large Mammalian Model for Extrahepatic Langerhans Transplantation for the Treatment of Type 1 Diabetes
}

\author{
George Kenchadze ${ }^{1}$, Ivane abiatari ${ }^{2}$, Ekaterine Berishvili ${ }^{3}$, Anzor Tchavtchavadze ${ }^{4}$ \\ 1,2,3,4 Ilia State university Tbilisi Georgia
}

\begin{abstract}
Beta-cell replacement therapy, by transplantation of isolated islets of Langerhans offers a physiologic alternative to subcutaneous insulin injections in treatment of treatment of type 1 diabetes. Successful beta-cell replacement provides excellent metabolic control without severe hypoglycemic episodes, a clear advantage compared to intensive insulin therapy. Islet transplantation is currently performed into the liver via intraportal access. However, the intrahepatic site and the intraportal route are associated with suboptimal islet engraftment, due to the proinflammatory microenvironment represented by the liver and by the procoagulant activity of the intraportal location. It has been estimated that $>50 \%$ of the islet graft is lost at the time of the transplant procedure. This is exemplified by the fact that islets isolated from multiple donors, and thus multiple islet injections are required to achieve graft success. For this reason, the identification of alternative, extrahepatic islet graft implantation sites are considered as a priority in the field.

Based on our previous research where our team, headed by Professor Berishvili Bernie created a successful rodent model of transplantation of islets inserted into isolated venous sacs we have created a similar model in large mammals.

We demonstrated that pancreatic islets successfully engrafted in the isolated venous sac with potential ability to restore euglycemia in diabetic Dogs. Therefore, the isolated venous sac offers a new site for transplantation of pancreatic islets. This would be clinically beneficial as an alternative to intrahepatic islet transplantation.
\end{abstract}

Keywords: Islets, Pancreas, Intravascular transplantation. 


\section{Introduction}

Type 1 diabetes and beta-cell replacement therapies Insulin-dependent diabetes mellitus (IDDM) is a chronic disease caused by progressive autoimmune destruction of the insulinproducing B-cells and usually diagnosed in childhood (1). Exogenous insulin treatment forces patients with type 1 diabetes to a lifelong therapy of multiple daily injections, which not only reduces quality of life, but is also unable to perfectly control hyperglycemia with the consequent development of microangiopathic and macroangiopathic lesions and the ensuing development and progression of chronic complications (nephropathy, retinopathy, ischemic heart disease, peripheral vasculopathy). Evidence that strict metabolic control can halt, or even prevent the development of late complications is in line with the notion that hyperglycemia is a major causative factor of diabetic complications. Unfortunately, intensive insulin therapy requires substantial effort and compliance from the patient and is accompanied by a threefold increase in the risk of severe hypoglycemic episodes (2). The relative ineffectiveness of subcutaneous insulin therapy is mainly attributable to its inability to reproduce the physiological tight control of blood glucose levels.

Beta-cell replacement therapy, by transplantation of insulin-producing tissue (either as whole pancreas or isolated islets of Langerhans) offers a physiologic alternative to subcutaneous insulin injections if the risks of the intervention and of the associated immunosuppression are lower than those faced by diabetic patients under conventional treatment (3). Successful beta-cell replacement provides excellent metabolic control without severe hypoglycemic episodes, a clear advantage compared to intensive insulin therapy.

Pancreas transplantation is a well-established surgical procedure and has improved considerably, with 5-year survival rates $>70 \%$ (4). However, whole pancreas transplantation is a technically challenging procedure, associated with significant morbidity, and not available in the developing world.

Intraportal transplantation of pancreatic islets has become a promising option for the cure of insulin dependent diabetes since the establishment of an automated method of large-scale human islet isolation and the subsequent first report of short-term insulin independence in a diabetic patient (5). Further progress has been made since the turn of the century and the publication of the Edmonton protocol. This protocol combined a glucocorticoid-free immunosuppressive regimen, isolation of higher quality human islets, and transplantation of higher quantities of islets in order to achieve a critical endocrine mass, using multiple donors. This protocol abruptly increased the rate of insulin-independence from $10 \%$ to $80 \%$ at 1 year and represented a huge boost to the field at the time (6).

Unfortunately, while $80 \%$ of cases achieved insulin independence at 1 year, this figure decreased to $10 \% 5$ years after transplantation (7). Recent developments, mostly in the immunosuppressive regimens used have however been able to yield insulin independence rates of $50 \%$ at 5 years $(8,9)$, indicating that progress is rapidly ongoing and that functional results of islet of Langerhans transplantation were close to matching those of whole pancreas. 


\subsection{Problems and issues associated with intraportal islet transplantation}

One major issue in intraportal human islet transplantation is the need for multiple donors and repeated islet infusions in order to achieve those results, indicating that significant tissue loss occurs at the time of transplantation and beyond. Early islet loss is facilitated by multiple factors related to the cellular microenvironment at the site of implantation. In effect, the intraportal site has 6 several features that contribute to the loss of islets and that make the liver a less than ideal implantation site (10). The live ris rich in Küppfer cells exposing the islet graft to proinflammatory cytokines and chemokines, reactive oxygen species and direct cytotoxicity (11) ; contact between islets and portal blood induces a thrombotic/inflammatory reaction called Instant Blood-Mediated Inflammatory Reaction (IBMIR) that leads to activation of the coagulation and complement cascades, granulocyte and monocyte infiltration, and ultimately islet destruction $(12,13)$; devascularized isolated islets are exposed to a low oxygen tension environment (14); localized hepatic steatosis has been reported after intraportal islet transplantation, and may expose the graft to lipotoxicity (15); the inherently hyperglycemic environment and the relatively higher concentrations of drug metabolites in the liver may also contribute to islet damage by direct $\beta$-cell toxicity (16).

It has been estimated that $50-70 \%$ of islets are destroyed in the immediate post transplant period by either IBMIR or by other inflammatory phenomena (17). This immediate loss of islets not only means that an excess of islets must be transplanted in order to achieve the critical mass of glucose-sensitive insulin-secreting tissue, but also inevitably leads to increased antigenic material being released in the host which may prime the adaptive immune response (18).

\subsection{Large mammal models of islet of Langerhans isolation and transplantation}

Most experimental studies in the field of islet of Langerhans transplantation are performed in rodents for obvious reasons of ease and costs. However, rodents can hardly be considered a valid preclinical model, and many strategies successful in rodents are not as successful when upscaled to large mammal models, let alone to the clinic. Therefore, the identification of a favorable alternative islet implantation site in the rodent must be confirmed in a large mammal. The most commonly used and better described large mammal models of islet of Langerhans isolation and transplantation are non-human primates, the pig and the dog. Nonhuman primates are considered as the animal model the closest to humans. It is a well characterized model that has indeed been used for preclinical studies in the field $(28,29)$. However, ethical concerns and cost issues preclude the use of this model in most countries or institutions, and at best implies its utilization in very small series of animals. The pig is the large mammal the most commonly used for islet isolation. However, it is mostly utilized as an islet donor rather than recipient, and the rationale for isolating porcine islets lies in its identification as the most likely candidate for xenogeneic islet donation in the clinical setting in the future (29). Porcine islets are very different in histological structure to human islets, which renders their isolation technically very challenging, and models of porcine autologous or allogeneic transplantation not fully relevant to the human situation $(30,31)$. The dog has been used since many years in models of islet isolation and autologous and allogeneic transplantation. Due to their structure very similar to that of human islets, canine islet isolation protocols are remarkably similar to those in use for clinical human islet isolation, which renders dogs an ideal model for preclinical studies of islet transplantation in terms of feasibility, relevance of results and costs (32-34). 


\subsection{Preliminary Studies}

We hypothesized, that the isolated intravascular site could be an advantageous site for pancreatic islet transplantation, because endothelial cells forming the inner lining of blood vessels effectively maintain an anti-inflammatory and anti-coagulant intravascular environment, and produce a number of growth factors, cytokines and other substances that may affect not only the endothelial cells themselves, but also surrounding cells. Moreover, blood vessels are well vascularized through abundant vasa-vasorum and, importantly, the intravascular site can be easily accessed, which would permit islet transplantation as a minimally invasive procedure. Retransplantation could readily be carried out on multiple occasions. Our recent studies established the feasibility of transplanting isolated pancreatic islets into a venous space fashioned from the lumbar vein in rats. We established that isolated vsenous space continued to be supplied with blood along with the preservation of integrity of venous channels for the release of substances secreted by transplanted islets into the bloodstream. Transplantation of minimal mass of islets (350 islets) into the isolated venous sac restored normoglycemia in STZ-treated diabetic rats. Comparison with the currently utilized method of intraportal islet transplantation showed that this novel method of cell transplantation was superior for correcting hyperglycemia Transplanted islets were shown to survive, engraft and function indefinitely (over several months) in the venous space. Morphological studies revealed that islets transplanted in isolated venous sacs had normal morphology, and maintained expression of insulin. The quality of glucose metabolic control in rats that reached normoglycemia via intravascular islet transplantation 11 was similar to that of normal control rats (Fig. 1). Moreover, since transplantation of islets in animals with toxin-induced diabetes led to normalization of high blood glucose levels, we concluded that this approach was successful for disease correction.

Encouraged by these results, we performed to a preclinical study in dogs, which obviously has a potential to translate to the clinical practice. We believe that the proposed procedure is simple, efficient and safe. Transplantation of pancreatic islets into the isolated venous sac would prove a major step forward, be clinically applicable and eventually solve issues encountered with the intrahepatic islet transplantation, notably the need for multiple donors still required for functional success.

Figure 1: Figure description (TNR 10pt., centered, italics)

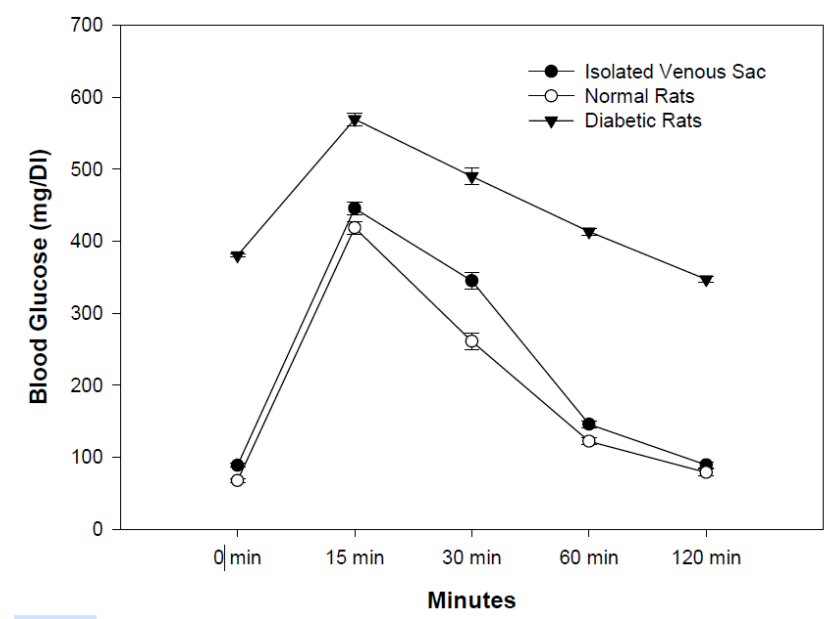


Fig 2 Fig. 1Glucose tolerance tests. Shows results in groups of animals challenged with intravenous glucose. Healthy rats showed normal glucose tolerance. In control untreated diabetic rats, glucose tolerance was impaired. By contrast, in rats treated with islet transplantation in venous sacs, glucose tolerance was normal

\section{Methods (TNR 14pt., bold)}

\section{Animals}

We used mongrel dogs weighing 12 to $17 \mathrm{Kg}$. Animals were housed in standard conditions under 12-hr day-night cycles with provision of animal meal and water ad libitum. Animals received care according to guidelines for Animal Experimentation at Ilia State University. All experiments were performed under protocols reviewed and approved by the institutional animal care and use ethical committee.

All surgical procedures were performed under general anesthesia, with a combination of Midazolam 0.1-0.2 mg/kg, Stresnil $0.5 \mathrm{mg} / \mathrm{kg}$ and Xylazine $2 \mathrm{mg} / \mathrm{kg}$. Endotracheal intubation along with insertion of a nasogastric tube for decompression was performed. Inhalation anesthesia was being achieved with 1.5 to $3 \%$ isofluorane. Propofol $3 \mathrm{mg} / \mathrm{kg}$ and Ketamine 3 $\mathrm{mg} / \mathrm{kg}$ was be administered through IV catherization as needed.

\section{Induction of Diabetes}

Diabetes was induced by total pancreatectomy. Briefly, the abdomen was entered through an upper vertical midline incision. After complete mobilization of the pancreas, the bile duct, was identified and in order to minimize ischemia and trauma to the duodenum, the duodenal branch of the gastroduodenal artery was identified and the pancreaticoduodenal arcade was resected. While leaving the major vascular connections intact, angiocatheters was introduced into the uncinate and corporeal pancreatic ducts before dissecting the pancreas from the duodenum. After pancreas removal, a heparinized silicon tube was secured in the portal vein, with the other end of the tube brought out under the skin and secured in a subcutaneous pocket for the collection of blood performed the consecutive days. The abdomen was irrigated with warm saline $2 \mathrm{X}$ and suctioned. After securing hemostasis, surgical wounds were sutured.

\section{Isolation of islets of Langerhans}

The islet isolation was performed using modified Ricordi's method with collagenase NB8 broad range (Serva; Uetersen, Germany) (32-34,68). Briefly, cannulated pancreatic ducts were manually infused with enzyme solution. After infusion, pancreas was cut into 8-10 pieces. Islet isolation were conducted in the Ricordi chamber filled with digestion solution at a temperature of $37^{\circ} \mathrm{C}$, with a heating circuit and gentle agitation. During the digestion process, $1 \mathrm{ml}$ samples were stained with dithizone to monitor dissociation under a microscope. Once free islets appear in the sample, the circuit was cooled, dilution with cold PBS has started and pancreatic digest was collected. Fetal bovine serum in concentration of $10 \%$ was used as an enzyme inhibitor. After three washes with HBSS containing $10 \%$ fetal bovine serum, tissue pellet was being resuspended in ice-cold University of Wisconsin solution and kept on ice for $30 \mathrm{~min}$. Islets were separated from acinar tissue on discontinuous Ficoll density gradients. Dissociated tissue were loaded in $1.090 \mathrm{~g} / \mathrm{ml}$ Ficoll and placed at the bottom of $250-\mathrm{ml}$ conical tubes, followed by layers of $1.077 \mathrm{~g} / \mathrm{ml}$ and $1.040 \mathrm{~g} / \mathrm{ml}$ Ficoll and 
centrifugated at $800 \mathrm{~g}$ for $5 \mathrm{~min}$. Islets were collected from the 1.077 and $1.040 \mathrm{~g} / \mathrm{ml}$ Ficoll interface. After two washes, islets were handpicked. Isolated islets were counted manually and viability was being determined by trypan blue dye exclusion. Purity of islets was verified by dithizone staining. Purified islets were cultured overnight free-floating $\left(37^{\circ} \mathrm{C}, 5 \% \mathrm{CO} 2\right)$ in culture medium consisting of CMRL 1066 supplemented with $2 \mathrm{mM} / \mathrm{L}$ L-glutamine, penicillin-streptomycin $(1000 \mathrm{U} / \mathrm{ml}-10 \mathrm{mg} / \mathrm{ml})$, and $10 \%$ (vol/vol) fetal bovine serum for 20 $24 \mathrm{~h}$ before transplants

\section{Results}

\subsection{Islet Quality Assessment}

More than 1400 islets were isolated from single donor, with more than 90 percent viability. The size of islets ranged from $50 \mu \mathrm{m}$ to greater than $500 \mu \mathrm{m}$ in diameter. Islet sizes ranged from 150 to $300 \mu \mathrm{m}$.

\subsection{Isolated Venous Sac}

No tributary to lumbar vane was disrupted during the venous sac preparation (Fig. 1). This procedure was well tolerated with no surgical morbidity or mortality.

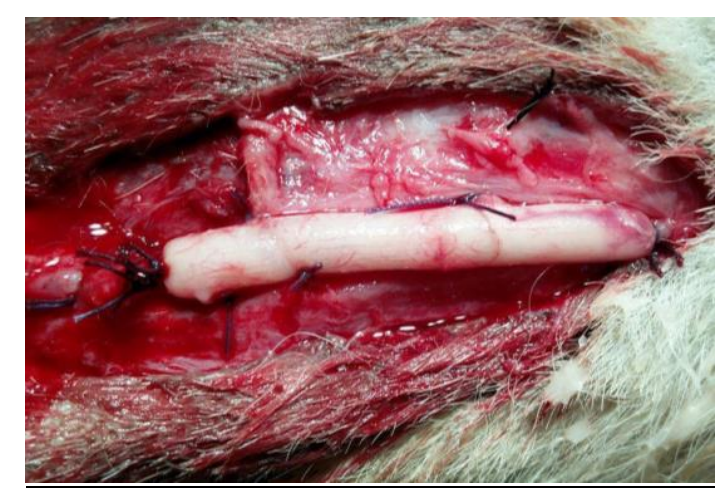

Fig. 1 isolated venous sac

\section{3 cell grafting and survival}

we observed that venus sac could contain up to 400 islets. Therefore, in all subsequent studies, we transplanted 350 islets. Histologic examination of islet graft-bearing venous sacs in rats demonstrated no inflammatory cell infiltration Transplanted islets seemed healthy and were embedded within connective tissue matrix (Fig. 2). We observed presence of venules and vasa vasorum and red blood cells in proximity to islets in venous sacs. 


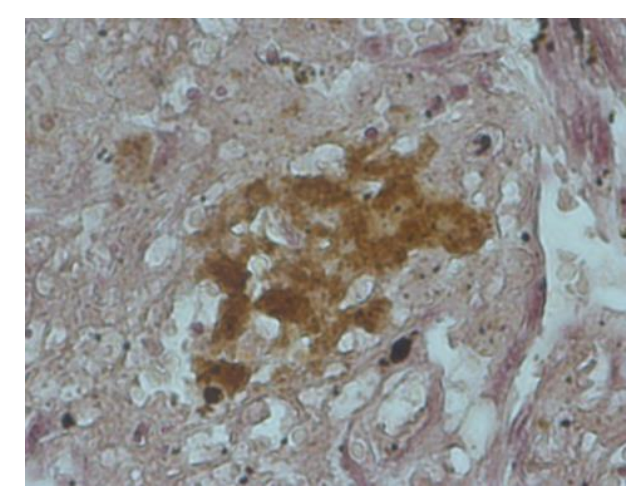

Fig. 2 : cells in isolated venous sac

\section{Discussion}

Transplantation of pancreatic islets into the liver is an acceptable and effective therapy for patients with type 1 diabetes mellitus and unstable glycemic control. Islet transplantation is far more attractive than the alternative of vascularized pancreas transplantation from the perspectives of surgical risk and recovery. However, the hepatic microenvironment is hostile to islet grafts. In the early loss of intraportally transplanted islets, contributors include low oxygen tension, cytokines/chemokines released by immunologically active cells, such as Kupffer cells (macrophages) (16), and instant blood-mediated inflammatory reaction $(17,18)$. Most experts agree appropriate microenvironments supporting islet engraftment will be very important for long-term viability and function of transplanted islets. Therefore, our identification of a more favorable endogenous environment for successful islet transplantation should be of considerable interest.

\section{Conclusion}

In conclusion, our model showed that the venous ensures engraftment and survival, transplanted pancreatic islets over the short term. This offers new avenues for cell therapy in type 1 diabetes. Moreover, the venous sac should be useful for testing the fate and function of stem cell-derived pancreatic beta cells or islets in the future. Other conditions may also be amenable to transplantation of cell types in the venous sac. The simplicity of transplanting islets in the venous sac should advance studies for clinical development. This will obviously need studies with transplantation of allogeneic islets. We do not anticipate that immunologic responses to allogeneic islets will be different in the venous sac versus in the portal vein. The significant divergences in the nature of animal and human allograft responses suggest that these studies will be most informative when allogeneic islets are transplanted in people.

\section{Acknowledgment}

This Paper Is an Output Of The SHOTA RUSTAVELI NATIONAL SCIENCE FOUNDATION OF GEORGIA Phd. Grant. 


\section{References}

Ricordi C. Islet transplantation: A brave new world. Diabetes. 2003; 52:1595. [PubMed: 12829621]

2. Ricordi C, Strom TB. Clinical islet transplantation: advances and immunological challenges. Nat

Rev Immunol. 2004; 4:259. [PubMed: 15057784]

3. Shapiro AM, Lakey JR, Ryan EA, et al. Islet transplantation in seven patients with type 1 diabetes

mellitus using a glucocorticoid-free immunosuppressive regimen. N Engl J Med. 2000; 343:230.

[PubMed: 10911004]

4. Harlan DM, Kenyon NS, Korsgren O, et al. Immunology of Diabetes Society. Current advances and

travails in islet transplantation. Diabetes. 2009; 58:2175. [PubMed: 19794074]

5. Vantyghem MC, Kerr-Conte J, Arnalsteen L, et al. Primary graft function, metabolic control, and

graft survival after islet transplantation. Diabetes Care. 2009; 32:1473. [PubMed: 19638525]

6. Bellin MD, Barton FB, Heitman A, et al. Potent induction immuno-therapy promotes longterm

insulin independence after islet transplantation in type 1 diabetes. Am J Transplant. 2012; 12:1576.

[PubMed: 22494609]

7. Hiller WF, Klempnauer J, Luck R, et al. Progressive deterioration of endocrine function after

intraportal but not kidney subcapsular rat islet transplantation. Diabetes. 1991; 40:134. [PubMed:

2015968]

Kakabadze et al. Page 7

Transplantation. Author manuscript; available in PMC 2013 August 27.

NIH-PA Author Manuscript NIH-PA Author Manuscript NIH-PA Author Manuscript

8. Scharp DW, Marchetti P, Swanson C, et al. The effect of transplantation site and islet mass on longterm survival and metabolic and hormonal function of canine purified islet autografts. Cell

Transplant. 1992; 1:245. [PubMed: 1344296]

9. Mattsson G, Jansson L, Carlsson PO. Decreased vascular density in mouse pancreatic islets after

transplantation. Diabetes. 2002; 51:1362. [PubMed: 11978631] 
10. Carlsson PO, Palm F, Andersson A, et al. Chronically decreased oxygen tension in rat pancreatic

islets transplanted under the kidney capsule. Transplantation. 2000; 69:761. [PubMed: 10755523]

11. Kakabadze Z, Gupta S, Brandhorst D, et al. Long-term engraftment and function of transplanted

pancreatic islets in vascularized segments of small intestine. Transpl Int. 2011; 24:175. [PubMed:

20819197]

12. Echeverri GJ, McGrath K, Bottino R, et al. Endoscopic gastric submucosal transplantation of islets

(ENDO-STI): technique and initial results in diabetic pigs. Am J Transplant. 2009; 9:2485.

[PubMed: 19775318]

13. Caiazzo R, Gmyr V, Hubert T, et al. Evaluation of alternative sites for islet transplantation in the

minipig: interest and limits of the gastric submucosa. Transplant Proc. 2007; 39:2620. [PubMed:

17954193]

14. Kriz J, Jirak D, Vilk GJ, et al. Vascularization of artificial beds for pancreatic islet transplantation

in a rat model. Transplant Proc. 2010; 42:2097. [PubMed: 20692417]

15. Pileggi A, Molano RD, Ricordi C, et al. Reversal of diabetes by pancreatic islet transplantation into

a subcutaneous, neovascularized device. Transplantation. 2006; 81:1318. [PubMed: 16699461]

16. Bottino R, Fernandez LA, Ricordi C, et al. Transplantation of allogeneic islets of Langerhans in the

rat liver: effects of macrophage depletion on graft survival and microenvironment activation.

Diabetes. 1998; 47:316. [PubMed: 9519734]

17. Bennet W, Sundberg B, Groth CG, et al. Incompatibility between human blood and isolated islets

of Langerhans: a finding with implications for clinical intraportal islet transplantation? Diabetes.

1999; 48:1907. [PubMed: 10512353]

18. Moberg L, Johansson H, Lukinius A, et al. Production of tissue factor by pancreatic islet cells as a

trigger of detrimental thrombotic reactions in clinical islet transplantation. Lancet. 2002; 360:2039. 